\title{
FOURTH ORDER NONLINEAR EVOLUTION EQUATIONS FOR GRAVITY-CAPILLARY WAVES IN THE PRESENCE OF A THIN THERMOCLINE IN DEEP WATER
}

\author{
SUMA DEBSARMA ${ }^{1}$ and K. P. DAS ${ }^{1}$
}

(Received 23 March 1999)

\begin{abstract}
For a three-dimensional gravity capillary wave packet in the presence of a thin thermocline in deep water two coupled nonlinear evolution equations correct to fourth order in wave steepness are obtained. Reducing these two equations to a single equation for oblique plane wave perturbation, the stability of a uniform gravity-capillary wave train is investigated. The stability and instability regions are identified. Expressions for the maximum growth rate of instability and the wavenumber at marginal stability are obtained. The results are shown graphically.
\end{abstract}

\section{Introduction}

Coherent interactions of two surface waves and one internal wave have been investigated by Ball [1], Thorpe [24], Watson, West and Cohen [25] and others. Olbers and Herterich [21] have given a mechanism for the generation of internal waves through their coupling with surface waves, in which they used the theoretical model of Hasselman [14] for incoherent three wave interaction. The resonant interaction between long internal waves and surface waves has been studied by Funakoshi and Oikawa [11] and $\mathrm{Ma}$ [19]. The problem of the influence of internal waves on surface waves was investigated by Das [4], Rizk and Ko [22] and very recently by Bhattacharyya and Das [2]. In [2], the authors studied the nonlinear evolution of a three-dimensional surface gravity wave packet in the presence of a thin thermocline. They also derived fourth order nonlinear evolution equations to study the stability of a uniform surface gravity wave train. Dysthe [9] first showed that a fourth order nonlinear evolution equation is a good starting point for the study of the stability of surface waves in deep

\footnotetext{
'Department of Applied Mathematics, University of Calcutta, 92 A. P. C. Road, Calcutta 700 009, India. (C) Australian Mathematical Society 2002, Serial-fee code 1446-1811/02
} 
water. Since then many authors $[3,6-8,12,13,15,16,23]$ have derived fourth order non-linear evolution equations in different contexts.

A study, similar to that made by Bhattacharyya and Das [2] for surface gravity waves, is made in the present paper for gravity-capillary waves in the presence of a thin thermocline. Two coupled nonlinear evolution equations correct to fourth order in wave steepness are derived for gravity-capillary waves. Assuming that the space variation of the amplitudes takes place along a line making an arbitrary fixed angle with the direction of propagation of the wave packet, the two coupled equations are reduced to a single equation. This single nonlinear evolution equation is employed in studying the stability of a uniform gravity-capillary wave train. The instability conditions are deduced. It is found that there exists instability for sufficiently short and sufficiently long waves. The regions of instability are shown in Figure 1. Expressions are obtained for the maximum growth rate of instability and for the wave number at marginal stability. Both these expressions have been plotted against wave steepness for different values of thermocline depth and perturbation angle. In the long wavelength region of instability, the maximum growth rate of instability is found to decrease with an increase in thermocline depth. But this effect is reversed in the short wavelength region of instability with very little increase in thermocline depth.

The paper is organised as follows. Basic equations are given in Section 2. The evolution equations are derived in Section 3. Stability of a uniform wave train is investigated in Section 4. Finally a conclusion is given in Section 5.

\section{Basic equations}

Following Bhattacharyya and Das [2] the velocity potential $\phi$ and free surface elevation $\zeta$ are broken up into two parts as follows:

$$
\phi=\phi_{0}+\phi_{1}, \quad \zeta=\zeta_{0}+\zeta_{1}
$$

where $\left(\phi_{0}, \zeta_{0}\right)$ and $\left(\phi_{1}, \zeta_{1}\right)$ correspond to the internal and gravity-capillary waves respectively.

The following are the equations for $\phi_{1}$ and $\zeta_{1}$ in which we disregard the existence of the thermocline. Since capillary-gravity and internal waves interact nonlinearly, in the following we keep full expressions for $\phi$ and $\zeta$ in the nonlinear terms without breaking:

$$
\begin{aligned}
\nabla^{2} \phi_{1} & =0, \quad-\infty<z<\zeta, \\
\frac{\partial \phi_{1}}{\partial z}-\frac{\partial \zeta_{1}}{\partial t} & =\left(\vec{\nabla}_{h} \phi\right) \cdot\left(\vec{\nabla}_{h} \zeta\right), \quad \text { at } z=\zeta,
\end{aligned}
$$




$$
\begin{aligned}
& \frac{\partial \phi_{1}}{\partial t}+g \zeta_{1}=-\frac{1}{2}(\vec{\nabla} \phi)^{2}+v\left[\frac{\partial^{2} \zeta}{\partial x^{2}}\left(1+\left(\frac{\partial \zeta}{\partial x}\right)^{2}\right)^{-3 / 2}\right. \\
&\left.+\frac{\partial^{2} \zeta}{\partial y^{2}}\left(1+\left(\frac{\partial \zeta}{\partial y}\right)^{2}\right)^{-3 / 2}\right] \quad \text { at } z=\zeta \\
& \frac{\partial \phi_{1}}{\partial z} \rightarrow 0, \quad \text { as } z \rightarrow-\infty
\end{aligned}
$$

Here $\vec{\nabla}_{h}$ is the horizontal gradient operator, $g$ is the acceleration due to gravity and $v$ is the ratio of the surface tension $T$ to the water density.

Assuming that the thermocline is confined between two planes $z=-d+\delta$ and $z=-d-\delta$, where the thermocline thickness $2 \delta$ is small, the equation for $\phi_{0}$ and $\phi_{0}^{\prime}$, the velocity potentials above and below the thermocline respectively, can be written as follows [10]. The terms responsible for long wavelength internal waves are assumed to be sufficiently small and so their product and higher degree terms have been neglected [10]:

$$
\begin{gathered}
\nabla^{2} \phi_{0}=0, \quad-d+\delta<z<\zeta, \\
\nabla^{2} \phi_{0}^{\prime}=0, \quad-\infty<z<-d-\delta, \\
\left(\partial \phi_{0} / \partial z\right)_{z=-d+\delta}=\left(\partial \phi_{0}^{\prime} / \partial z\right)_{z=-d-\delta}, \\
\frac{\partial^{2}}{\partial t^{2}} \nabla^{2} w+N^{2}(z) \nabla_{h}^{2} w=0, \quad-d-\delta<z<-d+\zeta, \\
\partial \phi_{0}^{\prime} / \partial z \rightarrow 0, \quad \text { as } z \rightarrow-\infty, \\
\left(\frac{\partial \phi_{0}}{\partial z}\right)_{0}-\frac{\partial \zeta_{0}}{\partial t}=\left(\vec{\nabla}_{h} \phi\right) .\left(\vec{\nabla}_{h} \zeta\right), \quad \frac{\partial \phi_{0}}{\partial t}+g \zeta_{0}=-\frac{1}{2}(\vec{\nabla} \phi)^{2},
\end{gathered}
$$

where $N^{2}(z)=-(g d \rho / d z) / \rho$ is the square of the Brunt-Vaisala frequency. In deriving (3) two more realistic assumptions have been made. These are the Boussinesq approximation and the neglect of nonlinear terms, the latter being due to the sufficiently small amplitude of long internal waves.

Since we assume that the thickness of the thermocline is small, (2) and (3) are replaced by equations obtained from them by approaching the limit $\delta \rightarrow 0$.

The equation obtained from (2) by approaching the limit $\delta \rightarrow 0$ is

$$
\left(\partial \phi_{0} / \partial z\right)_{z=-d}=\left(\partial \phi_{0}^{\prime} / \partial z\right)_{z=-d} .
$$

If we integrate (3) with respect to $z$ between the limits $-d-\delta$ to $-d+\delta$ and then proceed to the limit $\delta \rightarrow 0$ we get

$$
\left(\frac{\partial^{4} \phi_{0}}{\partial t^{2} \partial z^{2}}\right)_{z=-d}-\left(\frac{\partial^{4} \phi_{0}^{\prime}}{\partial t^{2} \partial z^{2}}\right)_{z=-d}=-g \frac{\delta \rho}{\rho} \nabla_{h}^{2}\left(\frac{\partial \phi_{0}}{\partial z}\right)_{z=-d}
$$


where $\delta \rho=\rho(-d-0)-\rho(-d+0)$ is the density increase through the thermocline.

\section{Derivation of evolution equations}

Following the standard multiple scale method we expand $\phi$ and $\zeta$ given in (1) as follows [4]:

$$
\phi=\phi_{0}+\sum_{n=1}^{\infty}\left(\phi_{n} e^{i n \psi}+\phi_{n}^{*} e^{-i n \psi}\right), \quad \zeta=\zeta_{0}+\sum_{n=1}^{\infty}\left(\zeta_{n} e^{i n \psi}+\zeta_{n}^{*} e^{-i n \psi}\right),
$$

where an asterisk denotes the complex conjugate; $\phi_{0}, \phi_{0}^{\prime}, \phi_{n}, \phi_{n}^{*}$ are functions of $\xi=\epsilon\left(x-C_{g} t\right), \eta=\epsilon y, \tau=\epsilon^{2} t, z ; \zeta_{0}, \zeta_{n}, \zeta_{n}^{*}$ are functions of $\xi, \eta, \tau ; \psi=k x-\omega t$. Here $\epsilon$ is a small parameter which measures the slowness of space-time variation of the amplitude of different harmonics of the wave; $\omega, k$ satisfy the following linear dispersion relation of the gravity capillary wave and $C_{g}=d \omega / d k$ is the group velocity

$$
\omega^{2}=g k+\nu k^{3} \text {. }
$$

As in Bhattacharyya and Das [2] we get the following solutions for $\phi_{n}, \phi_{0}, \phi_{0}^{\prime}$ :

$$
\begin{gathered}
\phi_{n}=\exp \left(z \Delta_{n}\right) A_{n}, \quad n=1,2, \ldots, \\
\bar{\phi}_{0}=\bar{A}_{0} e^{\epsilon \bar{k} z}+\bar{B}_{0} e^{-\epsilon \bar{k} z}, \quad \bar{\phi}_{0}^{\prime}=\bar{C}_{0} e^{\epsilon \bar{k} z},
\end{gathered}
$$

where $A_{n}$ is a function of $\xi, \eta, \tau ; \overline{A_{0}}, \bar{B}_{0}, \bar{C}_{0}$ are functions of $k_{\xi}, k_{n}, \tau ; \bar{k}=\sqrt{k_{\xi}^{2}+k_{\eta}^{2}}$; $\Delta_{n}$ is the operator

$$
\Delta_{n}=\left[\left(n k-i \epsilon \frac{\partial}{\partial \xi}\right)^{2}-\epsilon^{2} \frac{\partial^{2}}{\partial \eta^{2}}\right]^{1 / 2}
$$

operating on $A_{n}$; and $\bar{\phi}_{0}$ and $\bar{\phi}_{0}^{\prime}$ are the Fourier transforms of $\phi_{0}$ and $\phi_{0}^{\prime}$ respectively given by

$$
\left(\bar{\phi}_{0}, \bar{\phi}_{0}^{\prime}\right)=\frac{1}{(\sqrt{2 \pi})^{2}} \iint_{-\infty}^{\infty}\left(\phi_{0}, \phi_{0}^{\prime}\right) e^{i\left(k_{\xi} \xi+k_{n} \eta\right)} d \xi d \eta .
$$

Perturbation expansions of $A_{n}, \zeta_{n}, \bar{E}=\overline{A_{0}}+\bar{B}_{0}, \bar{F}=\overline{A_{0}}-\bar{B}_{0}$ and $\zeta_{0}$ are as follows ([4]):

$$
A_{n}=\sum_{j=n}^{\infty} \epsilon^{j} A_{n j}, \quad \zeta_{n}=\sum_{j=n}^{\infty} \epsilon^{j} \zeta_{n j}, \quad n \geq 1,
$$




$$
(\bar{E}, \bar{F})=\sum_{j=n}^{\infty} \epsilon^{j}\left(\bar{E}_{j}, \bar{F}_{j}\right), \quad \zeta_{0}=\sum_{j=n}^{\infty} \epsilon^{j} \zeta_{0 j} .
$$

The rest of the derivation process for the nonlinear evolution equations is similar to that of Bhattacharyya and Das [2]. So following this procedure the following two coupled nonlinear evolution equations are obtained for a gravity-capillary wave packet in the presence of a thin thermocline:

$$
\begin{gathered}
i \frac{\partial \zeta}{\partial \tau}+C_{1} \frac{\partial^{2} \zeta}{\partial \xi^{2}}+C_{2} \frac{\partial^{2} \zeta}{\partial \eta^{2}}+i C_{3} \frac{\partial^{3} \zeta}{\partial \xi^{3}}+i C_{4} \frac{\partial^{3} \zeta}{\partial \xi \partial \eta^{2}} \\
=d_{1} \zeta^{2} \zeta^{*}+i d_{2} \zeta \zeta^{*} \frac{\partial \zeta}{\partial \xi}+i d_{3} \zeta^{2} \frac{\partial \zeta^{*}}{\partial \xi}+\frac{1}{2} \zeta \frac{\partial E}{\partial \xi} \\
-\frac{\partial^{2} E}{\partial \xi^{2}}+4 \Gamma \bar{d} M\left(\frac{\partial^{2}}{\partial \xi^{2}}+\frac{\partial^{2}}{\partial \eta^{2}}\right) E=-16 \Gamma M \frac{\partial}{\partial \xi}\left(\zeta \zeta^{*}\right)-4 \frac{\partial}{\partial \xi} H \frac{\partial}{\partial \xi}\left(\zeta \zeta^{*}\right),
\end{gathered}
$$

where $\zeta=\zeta_{11}+\epsilon \zeta_{12}, E=E_{1}+\epsilon E_{2}, H$ is the two dimensional version of the Hilbert transform given by

$$
H \psi=\frac{1}{2 \pi} \iint_{-\infty}^{\infty} d \xi^{\prime} d \eta^{\prime} \frac{\xi^{\prime}-\xi}{r^{3}} \psi\left(\xi^{\prime}, \eta^{\prime}\right), \quad r=\left[\left(\xi^{\prime}-\xi\right)^{2}+\left(\eta^{\prime}-\eta\right)^{2}\right]^{1 / 2},
$$

$\Gamma=\delta \rho / \rho, \bar{d}=k d$ and the coefficients $C_{j}, d_{j}, M$ are given by

$$
\begin{aligned}
C_{1} & =\frac{3 m^{2}+6 m-1}{8(1+m)^{2}}, & C_{2} & =\frac{1+3 m}{4(1+m)}, \\
C_{3} & =-\frac{(1-m)\left(1+6 m+m^{2}\right)}{16(1+m)^{3}}, & C_{4} & =\frac{3+2 m+3 m^{2}}{8(1+m)^{2}}, \\
d_{1} & =\frac{8+m+2 m^{2}}{4(1+m)(1-2 m)}, & d_{2} & =\frac{3\left(4 m^{4}+4 m^{3}-9 m^{2}+m-8\right)}{4(1+m)^{2}(1-2 m)^{2}}, \\
d_{3} & =-\frac{(1-m)\left(8+m+2 m^{2}\right)}{8(1+m)^{2}(1-2 m)}, & M & =\frac{1+m}{(1+3 m)^{2}}, \quad m=\frac{v k^{2}}{g} .
\end{aligned}
$$

The two evolution equations (4) and (5) have been made dimensionless by introducing the following dimensionless quantities in primes and then finally dropping the primes:

$$
\tau^{\prime}=\omega \tau, \quad \xi^{\prime}=k \xi, \quad \eta^{\prime}=k \eta, \quad E^{\prime}=\left(2 k^{2} / \omega\right) E .
$$

In the absence of a thermocline (5) reduces to

$$
\frac{\partial E}{\partial \xi}=4 H \frac{\partial}{\partial \xi}\left(\zeta \zeta^{*}\right)
$$


Eliminating $\partial E / \partial \xi$ between (4) and (6) we get a single nonlinear evolution equation for a gravity-capillary wave packet in deep water in the absence of a thermocline, and this equation when compared with (2.20) of Hogan [15] provides a check on our computation.

Since the coefficients $d_{1}, d_{2}, d_{3}$ of the evolution equation (4) contain the factor $1-2 m$ in their denominators, the two coupled evolution equations (4) and (5) do not remain valid when $m=1 / 2$. This corresponds to the second harmonic resonance, which was considered in detail by McGoldrick [20].

Now to reduce the two coupled equations (4) and (5) to a single equation we assume the space variation of the amplitudes takes place in a direction making an arbitrary angle $\theta$ with the direction of propagation of the wave. We then transform the horizontal co-ordinates $\xi, \eta$ into the new co-ordinates $\xi^{\prime}, \eta^{\prime}$ according to the relations

$$
\xi^{\prime}=\xi \cos \theta+\eta \sin \theta, \quad \eta^{\prime}=-\xi \sin \theta+\eta \cos \theta,
$$

and then assume that $\zeta, E$ depend on $\xi^{\prime}$ only and not on $\eta_{i}^{\prime}$. Then following the same procedure as in Bhattacharyya and Das [2], we arrive at the following single nonlinear evolution equation for gravity capillary waves in deep water in the presence of a thin thermocline, where we drop primes on $\xi$ :

$$
\begin{aligned}
i \frac{\partial \zeta}{\partial \tau} & +\beta_{1} \frac{\partial^{2} \zeta}{\partial \xi^{2}}+i \beta_{2} \frac{\partial^{3} \zeta}{\partial \xi^{3}} \\
& =\Lambda_{1} \zeta^{2} \zeta^{*}+i \Lambda_{2} \zeta \zeta^{*} \frac{\partial \zeta}{\partial \xi}+i \Lambda_{3} \zeta^{2} \frac{\partial \zeta^{*}}{\partial \xi}+\Lambda_{4} \zeta H \frac{\partial}{\partial \xi}\left(\zeta \zeta^{*}\right)
\end{aligned}
$$

where

$$
\begin{aligned}
\Lambda_{1} & =d_{1}+\frac{8 \Gamma M \cos ^{2} \theta}{\cos ^{2} \theta-4 \Gamma \bar{d} M}, & \Lambda_{2} & =d_{2} \cos \theta, \\
\Lambda_{3} & =d_{3} \cos \theta, & \Lambda_{4} & =\frac{2 \cos ^{4} \theta}{\cos ^{2} \theta-4 \Gamma \bar{d} M}, \\
\beta_{1} & =C_{1} \cos ^{2} \theta+C_{2} \sin ^{2} \theta, & \beta_{2} & =C_{3} \cos ^{3} \theta+C_{4} \cos \theta \sin ^{2} \theta .
\end{aligned}
$$

Since $\cos ^{2} \theta-4 \Gamma \bar{d} M$ is a factor of the denominators of $\Lambda_{1}$ and $\Lambda_{4}$, the evolution equation (7) does not remain valid when $\cos ^{2} \theta-4 \Gamma \bar{d} M=0$. This is the resonance condition and is satisfied when the component of group velocity of the gravitycapillary wave along a line making an angle $\theta$ with the direction of the propagation wave becomes equal to the phase velocity of the long internal wave. These resonance curves in the $(m, \theta)$-plane for some different values of thermocline depths have been drawn in Figure 1. Further since the evolution equation (7) has been derived on the assumption that $d$ is finite, the evolution equation does not also remain valid for $\bar{d} \rightarrow \infty$. 


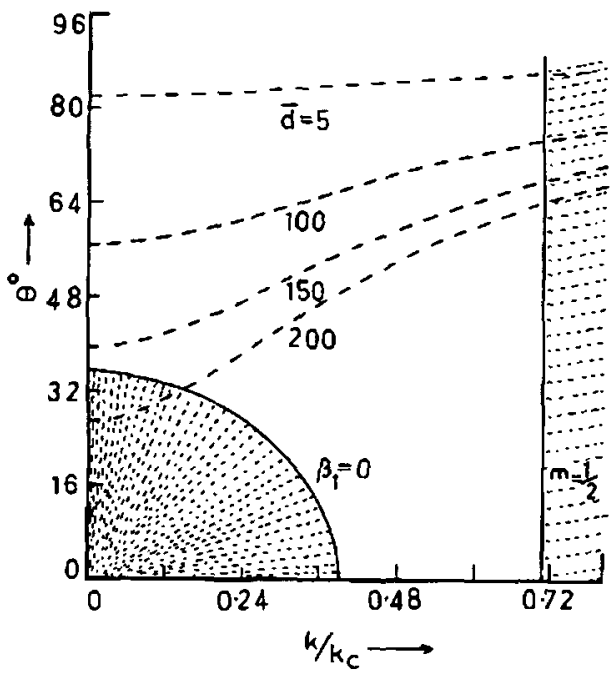

FIGURE 1. Regions of instability in the $(m, \theta)$-plane: instability occurs in the shaded areas. Resonance curves are indicated by a dashed line.

\section{Stability of uniform wave trains}

We consider the stability of a Stokes wave train taken in the form

$$
\zeta=\zeta^{(0)}=a_{0} e^{-i r \Delta \omega}
$$

which is a uniform wave train solution of the evolution equation (7). Consequently $\Delta \omega=\Lambda_{1} a_{0}^{2}$, where $a_{0}$ is a real constant and is the wave steepness. Thus $\Delta \omega$ is the amplitude dependent frequency-shift and it changes sign across $m=1 / 2$.

Now to make a stability analysis of the Stokes wave train, we consider the following perturbation of the uniform solution:

$$
\zeta=\zeta^{(0)}[1+\hat{\zeta}(\xi, \tau)]
$$

Substituting (8) into the evolution equation (7), linearizing with respect to $\hat{\zeta}, \hat{\zeta}^{*}$ and then separating into real and imaginary parts after setting $\hat{\zeta}=\hat{\zeta}_{r}+i \hat{\zeta}_{i}, \hat{\zeta}_{r}$ and $\hat{\zeta}_{i}$ being real, we arrive at the two equations for $\hat{\zeta}_{r}$ and $\hat{\zeta}_{i}$. Finally taking Fourier transforms of these two equations with respect to $\xi$, we get the following two equations:

$$
\begin{gathered}
-\frac{\partial\left(\hat{\zeta}_{i}\right)}{\partial \tau}+i\left(\beta_{2} l^{3}+\Lambda_{2} a_{0}^{2} l-\lambda_{3} a_{0}^{2} l\right)\left(\hat{\zeta}_{i}\right)+\left(-\beta_{1} l^{2}-2 \Lambda_{1} a_{0}^{2}+2 \Lambda_{4} a_{0}^{2}|l|\right)\left(\hat{\zeta}_{r}\right)=0, \\
-\beta_{1} l^{2}\left(\hat{\zeta}_{i}\right)+\frac{\partial\left(\hat{\zeta}_{r}\right)}{\partial \tau}-i\left(\beta_{2} l^{3}+\Lambda_{2} a_{0}^{2} l+\Lambda_{3} a_{0}^{2} l\right)\left(\hat{\zeta}_{r}\right)=0,
\end{gathered}
$$


where $\left(\hat{\zeta}_{r}\right)$ and $\left(\hat{\zeta}_{i}\right)$ denote Fourier transforms of $\hat{\zeta}_{r}$ and $\hat{\zeta}_{i}$ respectively defined by

$$
\left(\hat{\zeta}_{r, i}\right)=\frac{1}{\sqrt{2 \pi}} \int_{-\infty}^{\infty} \hat{\zeta}_{r, i} e^{-i l \xi} d \xi
$$

The following nonlinear dispersion relation is now obtained from (9) and (10) by assuming that the $\tau$ dependence of $\left(\hat{\zeta}_{r}\right)$ and $\left(\hat{\zeta}_{i}\right)$ is of the form $\exp (-i \Omega \tau)$ :

$$
\Omega=-\beta_{2} l^{3}-\Lambda_{2} a_{0}^{2} l \pm\left[\beta_{1} l^{2}\left(\beta_{1} l^{2}+2 \Lambda_{1} a_{0}^{2}-2 \Lambda_{4} a_{0}^{2}|l|\right)\right]^{1 / 2} .
$$

In this nonlinear dispersion relation order $\epsilon^{5}$ terms have been neglected, since the nonlinear evolution equation (7), from which this dispersion relation has been derived, is correct up to order $\epsilon^{4}$ terms.

The nonlinear dispersion relation (11) shows that there is instability if

$$
\beta_{1} l^{2}\left(\beta_{1} l^{2}+2 \Lambda_{1} a_{0}^{2}-2 \Lambda_{4} a_{0}^{2}|l|\right)<0,
$$

and this is possible when the wavenumber of perturbation satisfies the inequality

$$
l<l_{r}
$$

where

$$
l_{r}=a_{0} \sqrt{-2 \Lambda_{1} / \beta_{1}}+\Lambda_{4} a_{0}^{2} / \beta_{1} .
$$

In the case where there is instability, the maximum growth rate of instability is given by

$$
\gamma_{M}=a_{0}^{2}\left|\Lambda_{1}\right|\left(1-\frac{\Lambda_{4} a_{0}}{\Lambda_{1}} \sqrt{-\frac{\Lambda_{1}}{\beta_{1}}}\right)
$$

and this maximum occurs at the wavenumber of perturbation $l_{M}$ given by

$$
l_{M}=a_{0} \sqrt{-\Lambda_{1} / \beta_{1}}+3 \Lambda_{4} a_{0}^{2} / 4 \beta_{1} .
$$

From the expression of $l_{r}$ given by (12) we find that there is instability if $\Lambda_{1}$ and $\beta_{1}$ have opposite signs. In the $(\theta, m)$-plane $\beta_{1}$ changes sign accross the curve $\beta_{1}=0$ and due to the smallness of $\Gamma, \Lambda_{1}$ changes sign only across the line $m=1 / 2$. We can thus identify the regions in this plane where there are instabilities. These unstable regions in the $(\theta, m)$-plane when $m=\left(k / k_{c}\right)^{2}$ are shown in Figure 1. In this figure the curves $\cos ^{2} \theta-4 \Gamma \bar{d} M=0$ are drawn for some different values of thermocline depth $\bar{d}$. Our analysis of stability does not remain valid in the neighbourhood of these lines. For any particular value of $\theta$, we get two ranges of $m$, (i) $0<m<m_{0}$ and (ii) $m>1 / 2$ for which there are instabilities. Here $m_{0}$ depends on $\theta$. For $\theta=35.25^{\circ}, m_{0}=0$, 


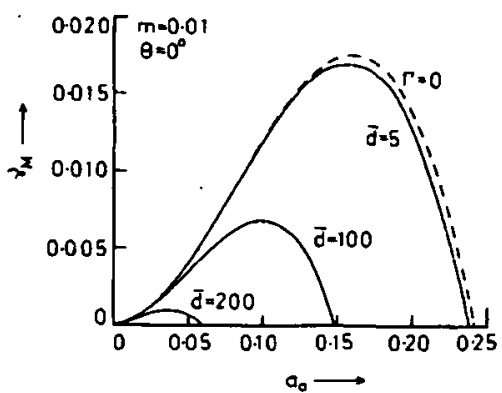

(a)

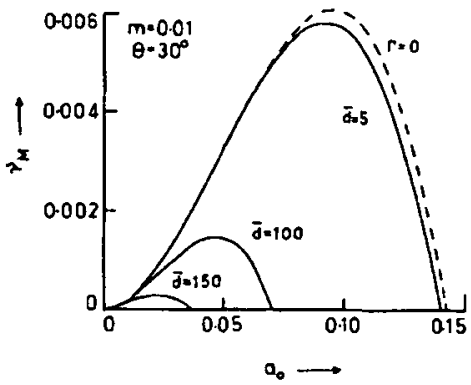

(c)

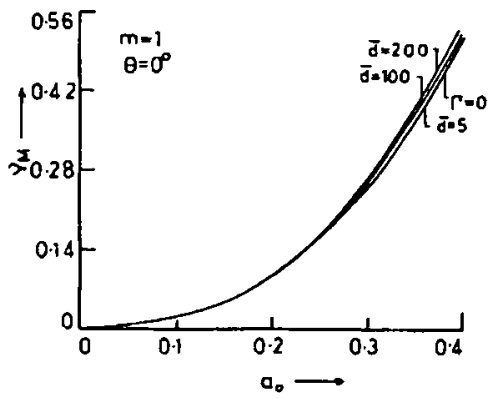

(e)

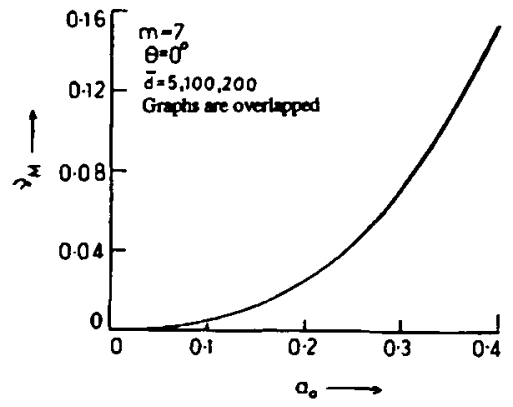

(g)

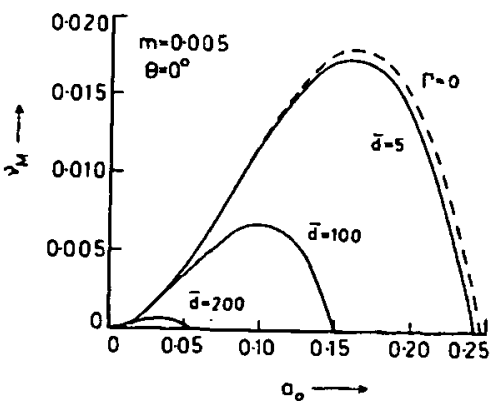

(b)

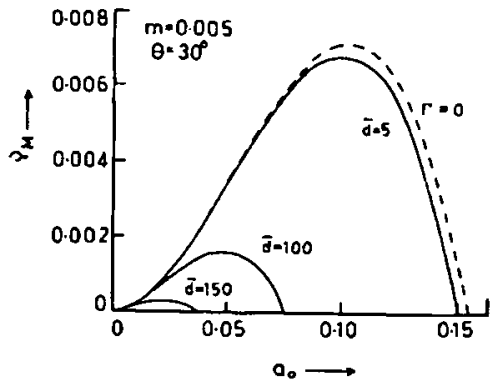

(d)

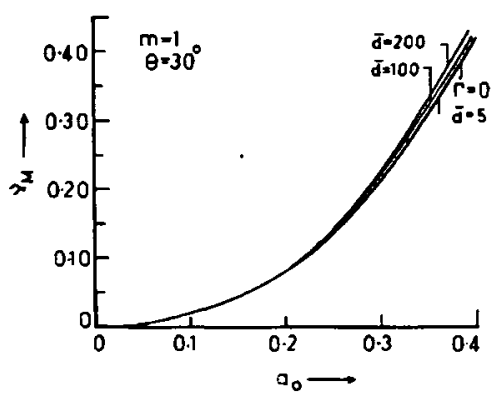

(f)

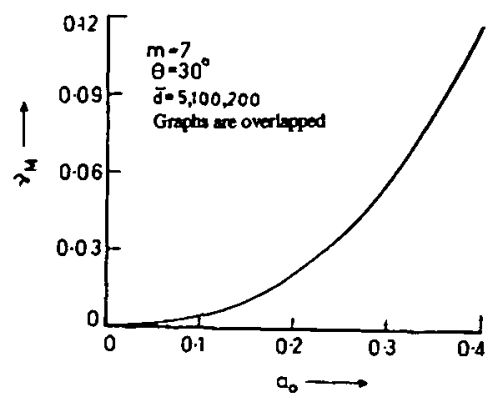

(h)

FIGURE 2. Maximum growth rate $\gamma_{M}$ against wave steepness $a_{0}$ for some values of $\theta, m$ and $\bar{d}$. 


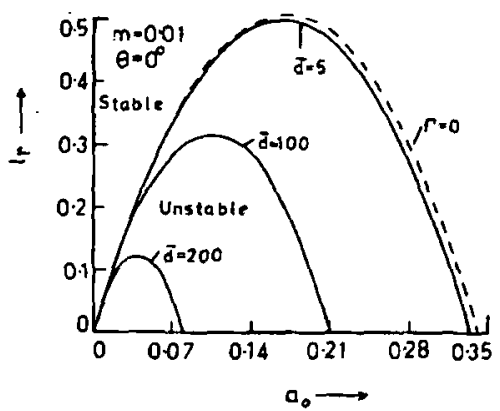

(a)

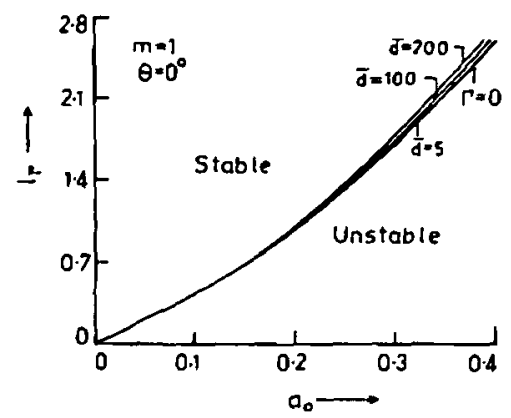

(c)

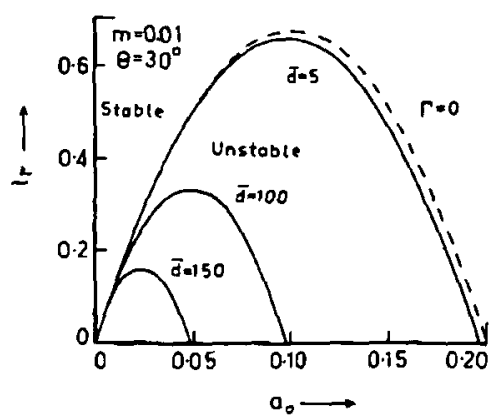

(b)

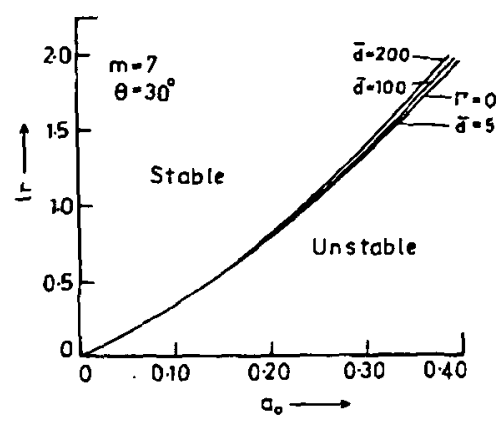

(d)

FIGURE 3. Wave number of perturbation $l_{r}$ at marginal stability against wave steepness $a_{0}$ for some values of $\theta, m$ and $\bar{d}$.

that is, for $\theta>35.25^{\circ}$, there is only one range of $m$ namely $m>1 / 2$ for which there is instability. For $\theta=0^{\circ}$, we easily find that $m_{0}=(2 / \sqrt{3}-1)$ as calculated by Hogan [15].

For numerical computation we have taken $\Gamma=10^{-3}$, which is the case for a seasonal thermocline. The maximum growth rate of instability $\gamma_{M}$ given by (13) has been plotted against wave steepness $a_{0}$ for some different values of $m, \theta, \bar{d}$. These are shown in Figures 2 (a)-(h). From these graphs it is seen that in the instability regions for which $0<m<m_{0}$, the growth rate of instability $\gamma_{M}$ decreases with an increase in thermocline depth $\bar{d}$. But in the instability region for which $m>1 / 2$, the increase in thermocline depth causes a slight increase in $\gamma_{M}$, that is, the thermocline depth has negligible influence on the growth rate of instability of sufficiently short gravity capillary waves.

We have also plotted the perturbation wave number $l_{r}$ at marginal stability given by (12) against the wave steepness $a_{0}$ for some diferent values of $m, \theta, \vec{d}$, showing stable-unstable regions in the $\left(a_{0}, l\right)$-plane. These are shown in Figures 3 (a)-(d). From these graphs we find that for comparatively long gravity-capillary waves for 
which $0<m<m_{0}$, the unstable region in the $\left(a_{0}, l\right)$-plane shrinks considerably with an increase in thermocline depth. But this effect is negligibly small and exactly opposite to that for sufficiently short waves for which $m>1 / 2$.

\section{Conclusion}

It has been pointed out by Dysthe [9] that a fourth order nonlinear evolution equation is a good starting point for the study of the stability of uniform wave trains in deep water since this gives results consistent with the exact results of Longuet-Higgins $[17,18]$. In view of this we have derived two coupled nonlinear evolution equations in order to study the stability of a uniform gravity-capillary wave train in deep water in the presence of a thin thermocline. These two coupled equations are reduced to a single equation under the assumption that the space variation of the amplitude takes place in an arbitrary fixed direction. This single equation does not remain valid (i) when the component of group velocity of the gravity-capillary wave along the direction of perturbation becomes equal to the phase velocity of the long wavelength internal wave and (ii) when there is a second harmonic resonance. From the single nonlinear evolution equation the instability condition for a uniform gravity-capillary wave train is derived. We find two regions of instabilty in the $(m, \theta)$-plane, where $m$ is the square of the ratio of $k$ (the wavenumber of the gravity-capillary wave) and $k_{c}=\sqrt{g / v}$ and $\theta$ is the angle made by the direction of perturbation with the direction of propagation of the gravity-capillary wave train. These two regions of instability have been shown in Figure 1. One of the two regions corresponds to waves of sufficiently short wavelength and the other corresponds to waves of sufficiently long wavelength. Both the maximum growth rate of instability and the wavenumber at marginal stability have been plotted against wave steepness $a_{0}$ for some different values of thermocline depth and $m$. The latter graphs give stable-unstable regions in the $\left(a_{0}, l\right)$-plane, where $l$ is the perturbation wavenumber. The unstable region in this plane is found to shrink with an increase in thermocline depth in the long wavelength region of instability. But this effect gets reversed in the short wavelength region of instability and is very little influenced by an increase in thermocline depth. Similar results are observed for the maximum growth rate of instability, as this is found to decrease with an increase in thermocline depth in the long wavelength region of instability. In the short wavelength region of instability an increase in thermocline depth causes a slight increase in the maximum growth rate of instability.

\section{References}

[1] F. K. Ball, "Energy transfer between external and internal waves", J. Fluid Mech. 19 (1964) $465-478$. 
[2] S. Bhattacharyya and K. P. Das, "Fourth order nonlinear evolution equations for surface gravity waves in the presence of a thin thermocline", J. Austral. Math. Soc. Ser. B 39 (1997) 214-229.

[3] U. Brinch-Nielsen and I. G. Jansson, "Fourth order evolution equations and stability analysis for Stokes waves on arbitrary water depth", Wave Motion 8 (1986) 455-472.

[4] K. P. Das, "On evolution equations for a three dimensional surface-gravity wave packet in a two layer fluid", Wave Motion 8 (1986) 191-204.

[5] A. Davey and K. Stewartson, "On three-dimensional packets of surface wave", Proc. Roy. Soc. London A338 (1974) 101-110.

[6] A. K. Dhar and K. P. Das, "A fourth order evolution equation for deep water surface gravity waves in the presence of wind blowing over water", Phys. Fluids A2 (1990) 778-783.

[7] A. K. Dhar and K. P. Das, "Fourth order nonlinear evolution equation for two Stokes wave trains in deep water", Phys. Fluids A3 (1991) 3031-3026.

[8] A. K. Dhar and K. P. Das, "Stability analysis from fourth order evolution equation for small but finite amplitude interfacial waves in the presence of a basic current shear", J. Austral. Math. Soc. Ser. B 35 (1994) 348-365.

[9] K. B. Dysthe, "Note on a modification to the nonlinear Schrödinger equation for application to deep water waves", Proc. Roy. Soc. London A369 (1979) 105-114.

[10] K. B. Dysthe and K. P. Das, "Coupling between surface wave spectrum and an internal wave: modulational interaction", J. Fluid Mech. 104 (1981) 483-503.

[11] M. Funakoshi and M. Oikawa, "The resonant interaction between a long gravity wave and a surface gravity wave packet", J. Phys. Soc. Japan 52 (1983) 1982.

[12] T. Hara and C. C. Mei, "Frequency downshift in narrowbanded surface waves under the influence of wind", J. Fluid Mech. 230 (1991) 429-477.

[13] T. Hara and C. C. Mei, "Wind effects on nonlinear evolution of slowly varying gravity-capillary waves", J. Fluid Mech. 267 (1994) 221-250.

[14] K. Hasselman, "Nonlinear interactions treated by method of theoretical physics (with application to generation of waves by wind)", Proc. Roy. Soc. London A229 (1967) 77.

[15] S. J. Hogan, "The fourth order evolution equation for deep water gravity-capillary waves", Proc. Roy. Soc. London A402 (1985) 359-372.

[16] P. A. E. M. Janssen, "On a fourth order envelope equation for deep water waves", J. Fluid Mech. 126 (1983) 1-11.

[17] M. S. Longuet-Higgins, "The instabilities of gravity waves of finite amplitude in deep water. I Super harmonics", Proc. Roy. Soc. London A360 (1978) 471-488.

[18] M. S. Longuet-Higgins, "The instabilities of gravity waves of finite amplitude in deep water. II Subharmonics", Proc. Roy. Soc. London A360 (1978) 489-505.

[19] Y. C. Ma, "A study of resonant interaction between internal and surface waves based on a two layer fluid model", Wave motion 5 (1983) 145.

[20] L. F. McGoldrick, "On Wilton's ripples: a special case of resonant interactions", J. Fluid Mech. 42 (1970) 193-200.

[21] D. J. Olbers and K. Herterich, "The special energy transfer from surface waves to internal waves", J. Fluid Mech. 92 (1979) 349-379.

[22] M. H. Rizk and D. R. S. Ko, "Interaction between small scale surface waves and large scale internal waves", Phys. Fluids 21 (1978) 1900-1907.

[23] M. Stiassnie, "Note on modified nonlinear Schrödinger equation for deep water waves", Wave Motion 6 (1984) 431-433.

[24] S. A. Thorpe, "On wave interaction in a stratified fluid", J. Fluid Mech. 24 (1966) 737.

[25] K. M. Watson, B. J. West and B. I. Cohen, "Coupling of surface and internal gravity waves: a mode coupling model", J. Fluid Mech. 77 (1976) 185. 